\title{
Measurement of the top-quark pair production cross-section using final states with an electron or a muon and a hadronically decaying $\tau$-lepton
}

\author{
Yuta Takahashi, On behalf of the ATLAS Collaboration \\ Graduate School of Science, Nagoya University, Furo-cho, Chikusa-ku, Japan
}

\begin{abstract}
A measurement of the top-quark pair production cross-section is reported using proton-proton collision data at $\sqrt{s}=7 \mathrm{TeV}$ recorded by ATLAS experiment at the Large Hadron Collider. Events are extracted from $2.05 \mathrm{fb}^{-1} \mathrm{of}^{\mathrm{s}}$ data by requiring an isolated electron or muon, a large missing transverse momentum, two or more energetic jets and a hadronically decaying $\tau$-lepton. The measured cross-section, $\sigma_{t \bar{t}}=186 \pm 13$ (stat.) \pm 20 (syst.) \pm 7 (lumi.) pb, is in good agreement with the Standard Model prediction.
\end{abstract}

Keywords:

Top quark, Cross section, $\tau$

\section{Introduction}

The top-quark ( $t$-quark) is the heaviest fundamental particle observed so far. Owing to its large mass, the $t$-quark has a strong connection with new physics such as the production via a $4^{\text {th }}$-generation quark or decay through a charged Higgs boson $\left(H^{ \pm}\right)$. To look for this effects, the production and the decay process of the $t$-quark need to be precisely understood through a measurement of the $t$-quark pair $(t \bar{t})$ production crosssection $\left(\sigma_{t \bar{t}}\right)$. The ATLAS Collaboration has measured $\sigma_{t \bar{t}}$ using the single-lepton $\left(t \bar{t} \rightarrow \ell^{+} v_{\ell} q q^{\prime} b \bar{b}, \ell=e, \mu\right)$ [1] and di-lepton $\left(t \bar{t} \rightarrow \ell^{+} v_{\ell} \ell^{-} \overline{v_{\ell}} b \bar{b}\right)$ [2] channels $(\ell=e, \mu)$, and found it to be consistent with the theoretical prediction $\left(164_{-16}^{+11} \mathrm{pb}\right)$. In these proceedings, first results on $\sigma_{t \bar{t}}$ are reported with $t$-quarks decaying to $\tau$ and either $e$ or $\mu$ [3] measured with the ATLAS detector [4]. This measurement is essential to search for the charged Higgs boson, as it is expected to decay more often into $\tau$-lepton than e or $\mu$.

\section{Event Selection}

In order to extract $t \bar{t}$ events, events are selected from $2.05 \mathrm{fb}^{-1}$ proton-proton collision data, by requiring one high transverse momentum $\left(p_{T}\right)$ lepton (an electron or a muon), two or more jets with $p_{T}>25 \mathrm{GeV}$ and at least one hadronically decaying $\tau$ candidate. The $\tau$ candidate is required to have $20 \mathrm{GeV}<p_{T}<100 \mathrm{GeV}$, to have $1\left(\tau_{1}\right)$ or $2,3\left(\tau_{3}\right)$ tracks with $p_{T}>1 \mathrm{GeV}$. The charge of the $\tau$ candidate is the sum of the charges of the associated tracks, and is required to be non-zero. In addition to this, missing transverse momentum of magnitude $E_{T}^{m i s s}>30 \mathrm{GeV}$ is required to reduce the multi-jet backgrund and the scalar sum of the $p_{T}$ of the leptons ( $e$ or $\mu$ and the $\tau$ candidate), jets and $E_{T}^{\text {miss }}$ is required to be greater than $200 \mathrm{GeV}$ to obtain $t \bar{t}$ enriched samples. Finally, at least one of the jets is required to be identified as originating from a $b$-quark to remove the $W(\rightarrow \ell v)+$ jet process.

The background arises from misidentifying a quark or gluon jet as a $\tau$ candidate. The $t \bar{t}$ single-lepton process is the dominant background as the final state topology is similar.

\section{Background modelings}

A hadronically decaying $\tau$-lepton typically generates a narrower, more collimated jet than one originating 
from a quark or gluon. A discriminant variable based on a Boosted Decision Tree (BDT) is constructed using 8 (11) parameters for $\tau_{1}\left(\tau_{3}\right)$. The similarity of the $\tau$ candidate to a real $\tau$-lepton is represented as a BDT output score, ranging from 0 (jet-like) to 1 ( $\tau$-like). A number of backgrounds can be estimated by exploiting the shape difference of the BDT output score.

A closer look at the background distribution reveals that it consists of mainly three components: light-flavor jets $(u, d, c, s)$, gluon jets and $b$-jets faking a $\tau$ candidate. Due to their narrow shower width, light-flavor jets tend to have a higher BDT compared to other jet types. Therefore, the estimation of the background is sensitive to the jet composition.

Based on this fact, events are split into opposite sign (OS) samples $\left(Q_{\tau} \times Q_{\ell}<0\right)$ and same sign (SS) samples $\left(Q_{\tau} \times Q_{\ell}>0\right)$ according to the charge correlation between the $\tau$ candidate and the lepton. The light-flavor components, especially the ones produced from $W$ boson (e.g, $t \bar{t}$ single-lepton or $W+$ jet) tend to be OS $>$ SS, while others tend to be OS $=\mathrm{SS}$. As for the $b$-jet components, it is produced as a pair $(b \bar{b})$ in the $t \bar{t}$ events and thus become OS $=\mathrm{SS}$. The gluon components do not initially have a charge and thus can be either OS and $\mathrm{SS}$ with same probability $(\mathrm{OS}=\mathrm{SS})$. Therefore, by performing OS - SS, $b$-jet and gluon components can be removed without losing any signal events enabling us to focus on the light-flavor backgrounds only.

\section{Identification of the events with real $\tau$-lepton}

Now that the background contains only light-flavor jets faking a $\tau$-lepton, events with a real $\tau$-lepton are identified by performing a template fit to the BDT output score with a signal and a background template.

The signal template is derived from $\tau$ candidates in the MC simulation that are truth-matched to a real $\tau$ lepton in the proportion expected from simulated events passing the event selection. $Z \rightarrow \tau \tau$ and diboson processes are also included as they include real $\tau$-leptons, which are removed after the fitting is performed. The background template is derived using a data control region with no $b$-jet, where the same event selections are applied except for $0 b$-jet requirement. These data are dominated by $W+$ jet events with similar kinematics to that of the backgrounds in the signal region. To be the light-flavor origin, the OS-SS subtraction is also performed on this sample. As the $0 b$-jet sample includes real $\tau$ contributions (mainly from $Z \rightarrow \tau \tau$ ), these are removed relying on the $\mathrm{MC}$ simulation.

Figure 1 shows the fitting result to the remaining events, where one can see the clear enhancement of the signal above the background, and Table 1 summarizes the results.

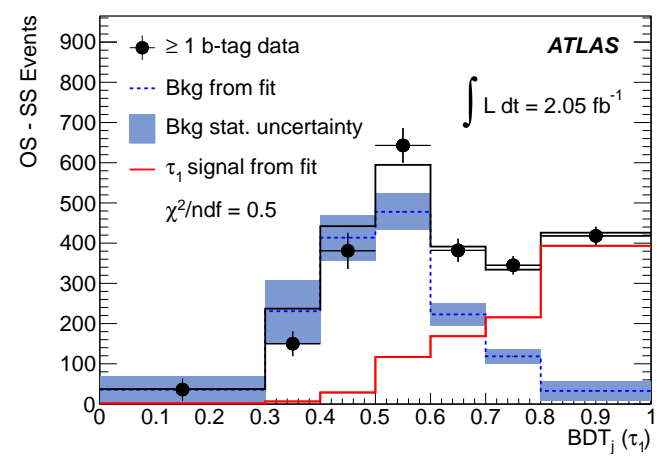

Figure 1: A fit result to the remaining events (combining the muon and the electron channel). The normalization is derived from a fit to the data. The fitted contributions are shown as the red line (signal), dotted blue line (background) and black (total) lines. The shaded blue bands are the statistical uncertainty of the background template [3].

\begin{tabular}{llll}
\hline & $\mathrm{N}_{\text {signal }}$ & $\mathrm{N}_{\exp }$ & $\sigma_{t \bar{t}}$ (stat, syst, lumi) \\
\hline$\mu+\tau_{1}$ & $445 \pm 43$ & 388 & $189 \pm 16 \pm 20 \pm 7 \mathrm{pb}$ \\
$e+\tau_{1}$ & $391 \pm 46$ & 338 & $190 \pm 20 \pm 20 \pm 7 \mathrm{pb}$ \\
$\mu+\tau_{3}$ & $125 \pm 33$ & 116 & $180 \pm 40 \pm 21 \pm 6 \mathrm{pb}$ \\
$e+\tau_{3}$ & $105 \pm 30$ & 101 & $170 \pm 50 \pm 21 \pm 6 \mathrm{pb}$ \\
\hline
\end{tabular}

Table 1: From left to right, the obtained number of signal events, expected number of signal events derived from MC simulation, and the obtained cross-section [3].

The combined cross-section for $e+\tau_{1}, \mu+\tau_{1}, e+\tau_{3}$, and $\mu+\tau_{3}$ yields $\sigma_{t \bar{t}}=186 \pm 13$ (stat.) \pm 20 (syst.) \pm 7 (lumi.) pb, which is consistent with the theoretical prediction $\left(164_{-16}^{+11} \mathrm{pb}\right)$. The result is the most precise measurement with a $\tau$-lepton final state performed so far with a relative uncertainty of $13 \%$.

\section{Conclusion}

The $t \bar{t}$ production cross-section is measured using proton-proton collision data at $\sqrt{s}=7 \mathrm{TeV}$ requiring a hadronically decaying $\tau$-lepton and a lepton (electron or muon) final state. The obtained cross-section is in good agreement with the Standard Model prediction.

\section{References}

[1] ATLAS Collaboration, ATLAS-CONF-2011-121

[2] ATLAS Collaboration, JHEP 1205 (2012) 059

[3] ATLAS Collaboration, Physics Letters B 717 (2012) 89-108

[4] ATLAS Collaboration, 2008 JINST 3 (2008) S08003 